\title{
An Application of Image Analysis to Hypersonic Flows
}

\author{
M. Mirzaei ${ }^{1}$, M. Belan ${ }^{1}$ \\ ${ }^{1}$ Department of Aerospace Engineering, Politecnico di Milano, Italy
}

\begin{abstract}
Image-based measurement methods are becoming increasingly common in fluid dynamics and are seen in applications ranging from low subsonic to hypersonic flows. This work is focused on the use of image processing methods for investigations of hypersonic jet flows, visualized through the electron beam technique. Indications about the jets propagation and their resulting morphologies are obtained by means of several techniques carried out on fluorescent images. The main topics presented are an improved measurement technique for moving structures velocities, an image processing method for denoising and reconstruction of jet images and an optical technique for measurements of the jet spreading angles.
\end{abstract}

\section{Introduction}

A wide range of visualization techniques have been developed over the years for understanding the physical phenomena of complicated 3D fows. Traditional visualizations were primarily qualitative, yielding limited information on flow structures; any quantification required laborious measurements, even by hand. An important achievement of modern experimental fluid mechanics is the invention and development of digital techniques for visualization and measurement of instantaneous flow velocity fields, flow structures, pattern formation and so on. In the most part of modern techniques, like particle tracer methods, the flow is illuminated in such a way as to make visible the flow particles in a finite domain. A series of images of the flow are captured to record how the particles move in response to the fluid flow and then the quantitative analyses of the flow, including velocities and structures characterizations, are performed by image processing which plays an ever-increasing role in fluid mechanics research. The best applied visualization method depends on the tests being performed and sometimes common methods do not work for particular tests and other innovative methods are used.

Hypersonic jet flow is one of these topics; it has been a widely studied subject over the past half century owing to its importance both in basic fluid dynamics and in applications for aeronautical and mechanical industries and also astrophysics. Over the last decades several experimental systems have been produced to create and visualize this kind of jets, and most of these experiments used innovative techniques for visualization. Some examples are the plasma jets created by laser ablation of shaped targets or with lasers colliding on aluminum foils $[1,2,3]$, the laboratory plasma jets which have been produced involving magneto-hydrodynamics effects [4], the laboratory study concerning the defection of supersonic jets produced by molecular clouds [5]. In general, these methods can visualize the jets only on short scale lengths. The apparatus used in the present work is completely different from mentioned ones, and built specif cally for the generation and visualization of hypersonic jets on long scales [6]. From the point of view of image analysis, the main principle of this system is the production of fluorescent images of the flow under test by an electron beam; the flow is then displayed and analyzed by image processing methods.

The next section presents briefly the experimental system and section 3 focuses on the image processing methods used to denoise and reconstruct images, then to measure moving structures velocities and jet spreading angles. Section 4 provides results of a sample jet showing how these methods can be applied. Finally, in section 5 we present our conclusions.

\section{Experimental system and working principle for Visualization}

In our experimental apparatus the jets are created by a fast piston system coupled with a de Laval nozzle chosen out of a set (detailed descriptions of the facility are available in reference [6]). The jet issuing from the nozzle propagates along the longitudinal axis of a cylindrical vacuum vessel. The visualizations are based on the electron beam method: the jet travels along the vessel and intersects an electron sheet. Here the fluorescence takes place and permits to acquire images. A high speed camera equipped with an image intensifier 
captures these images (Figure 1) and then the flow is analyzed by image processing. The present setup produces $512 \times 512$ monochromatic images at frame rates of 2000 to $8000 \mathrm{fps}$. This system has also the possibility to move the electron beam vertically to capture different 2-D sections from 3-D flow in each desirable vertical position. In our experiment the plane is set on the middle of jet flow.

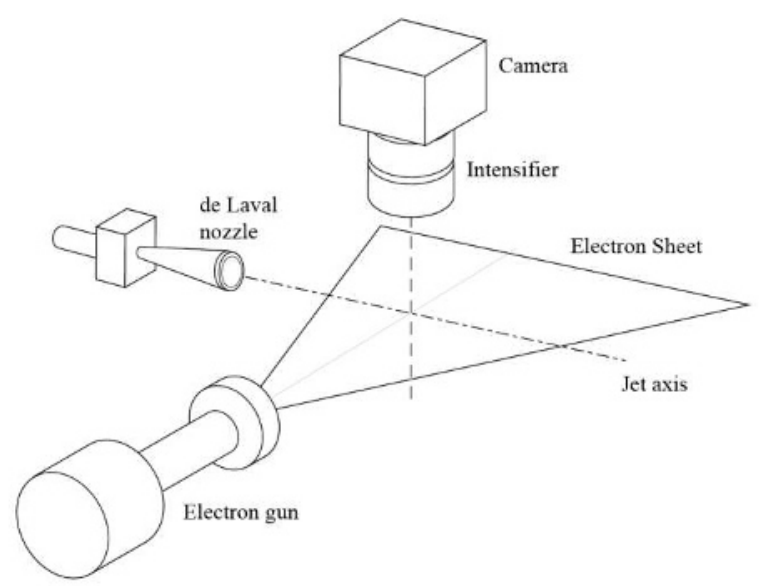

Fig. 1. Visualization system.
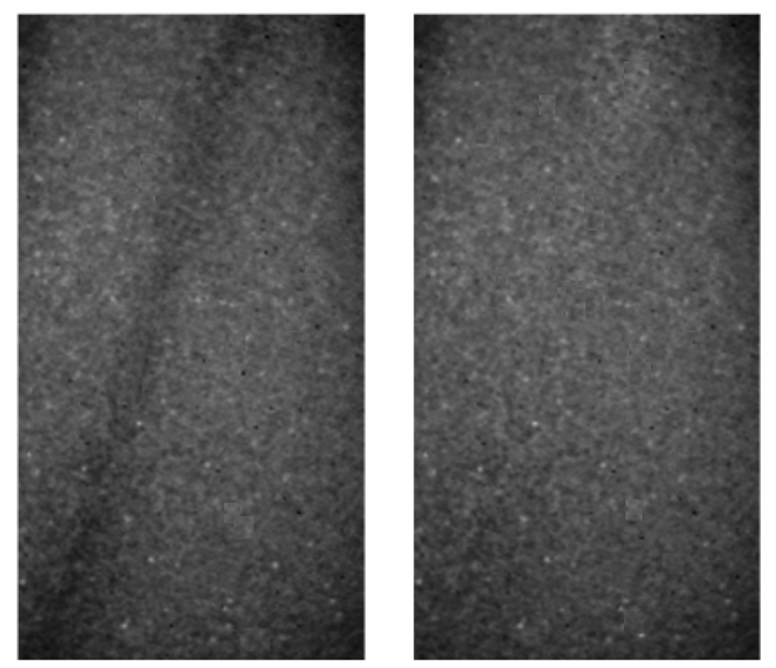

Fig. 2. Dark strip compensation

\section{Denoising and velocity measurement techniques}

Left panel of Figure 2 shows a sample of electron sheet image, without jet; an oblique dark strip exists in the captured plane because of low-response pixels on the CMOS sensor of camera causing an output level drop in a narrow area. This flaw can severely limit the quality of the images when the jet appears, and it is removed by a standard technique based on a multiplicative compensating function:

$$
f(i, j)=s(i, j) \times n(i, j)
$$

To obtain the desirable (uncorrupted) image denoted by $\mathrm{f}(\mathrm{i}, \mathrm{j})$, the compensating function, denoted $\mathrm{n}(\mathrm{i}, \mathrm{j})$, is multiplied by the acquired image denoted $s(i, j)$. As a first step, $\mathrm{n}$ is obtained as a two-dimensional function from a reference image, and then it is multiplied to all the other frames.

In our experiment, a cross-correlation technique is used to measure the structure velocities by determining the displacement of a structure moving through a pair of consecutive images. For example, consider two consecutive frames where the jet head appears. Extracting the middle rows from the matrices of the first and second image, two vectors $\mathrm{f}$ and $\mathrm{g}$ are obtained, containing the grey levels of the rows. These vectors differ roughly by an unknown shift along the x-axis. Practically, the most probable shift is often found by seeking the maximum of the correlation function

$$
(f * g)[n]=\sum_{m} f[m] \quad g[n+m]
$$

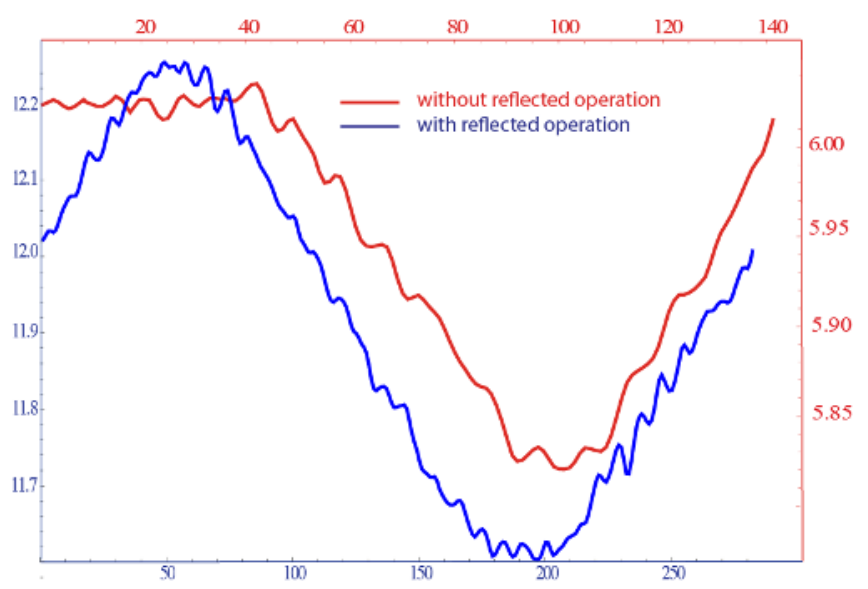

Fig. 3Integral correlation curves for two methods

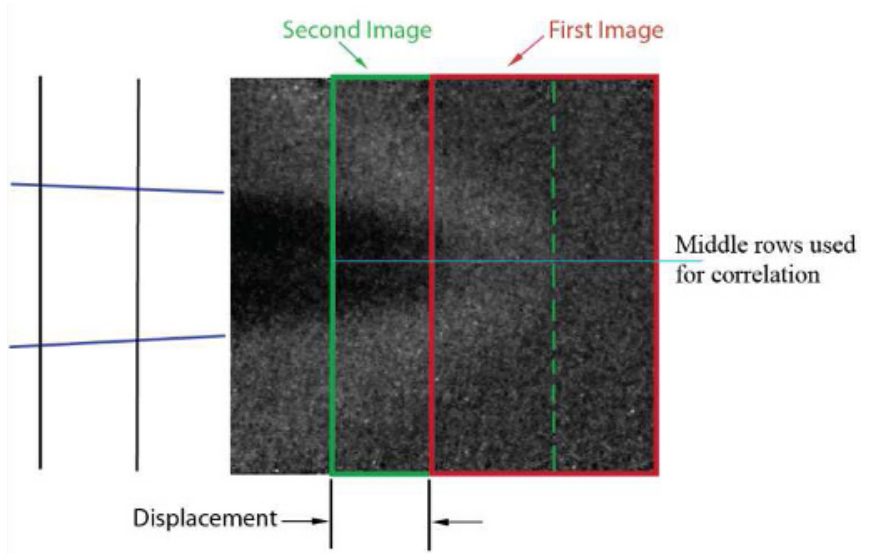

Fig. $.4 \square$ correlated superposition of original images 
Red curve of figure 2 shows the correlation for these two vectors and as is obvious the peak is not so certain, because the second vector is not just a translation of the first one: this causes a wide uncertainty on displacement. In order to reduce this uncertainty, the operation is modified by a cross-reflection technique; $\mathrm{f}$ is reflected with respect to the common origin, then a new vector $\mathrm{g}^{\prime}=(\mathrm{Rf}) \mathrm{U} \mathrm{g}$ (union of reflected $\mathrm{f}$ and $\mathrm{g}$ ) is created; the same process is applied to $g$, giving rise to $f^{\prime}=(R g) U f$. This leads to a new correlation curve $\mathrm{f}^{\prime}{ }^{*} \mathrm{~g}^{\prime}$ where the correlation peak is clearer (blue curve of figure 3 ). The position of the peak gives the shift between the two vectors or in other words the jet head displacement between two frames; the resulting velocity is simply obtained by dividing the displacement by the interframe time. Then by iterative superposition, a wider jet image is reconstructed (figure 4). This procedure can be iterated on a limited number of images for reasons of physical meaning, essentially to avoid a 'frozen' representation of the jet under study.

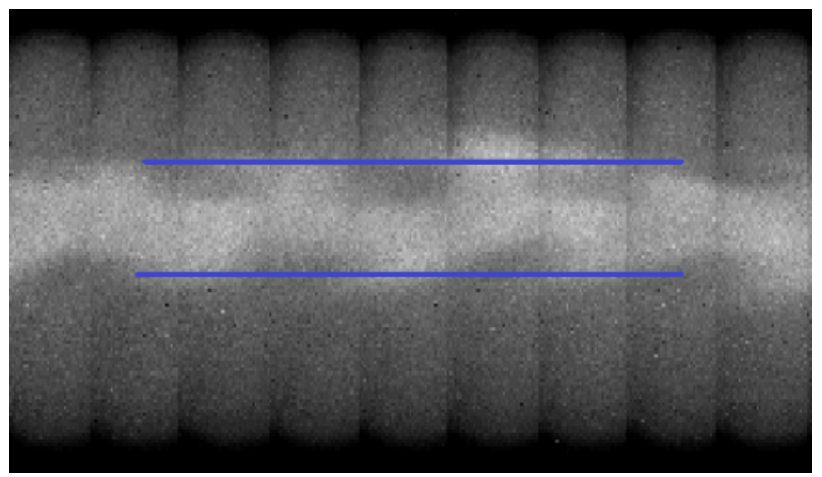

) ig. $\$ 5 \square$ selected rows for correlation measurement )

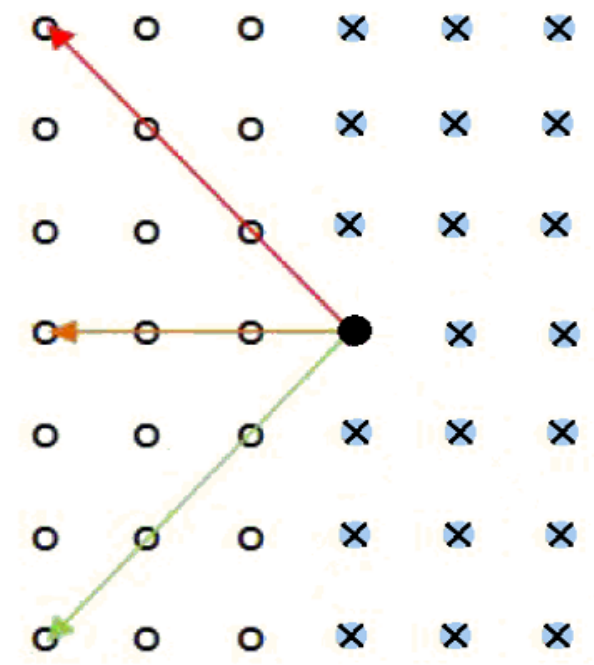

) ig.60 Considered pixels in directional median inpainting; o shows a known pixel, $\times$ shows an unknown pixel
The same technique is used also to study moving structures in the parts of the jet where flow has remarkable instabilities. In this case, the right image rows must be considered for a proper correlation. Figure 5 shows an example where two rows are selected on the jet boundaries where a supersonic Kelvin-Helmholtz (K-H) instability develops, here the technique is applied to both rows and the final displacement is obtained by average. Sometimes, the obtained displacement is not accurate, because there are 3-D instabilities rotating and changing shape along the jet. In this case different displacements in different locations must be correctly reported. Furthermore, the velocity of these instabilities varies along the jet and in the worst case it is so fast that jet structures do not have any overlap in two consecutive frames, so that the shift is greater than the frame width and cannot be measured. In other cases, the structures have shifts lower than the frame width but they fall on the border of the electron sheet: in these cases, the reconstructed image is not continuous and there are undetected region between frames. When these regions are small with respect to the jet diameter, inpainting technique has been used to fill the missed data using samples of the available image data in the neighboring pixels to reconstruct a continuous jet image. The used inpainting method is based on directional median filters applied by an iterative algorithm. In the first iteration, the median value of three known pixels in the boundary of undetected columns in three directions is calculated, and then the unknown pixel is replaced by the median of the obtained values (figure 6). This process, limited to three directions, let data propagate longitudinally from both sides of missed data so that the region under processing is filled. In the last iteration, this process is repeated in all directions and the obtained values are replaced. In our images, this algorithm goes for 3 or more iterations and provides adequate results to get continuous display.

The next process is filtering, applied to remove image noise in the final reconstructed image. Captured images are disturbed by some random fluctuations (figure 2 and 5). This noise is inherently due to the fluorescence phenomenon and to the accumulative nature of photon detection which depends on the number of photons captured in an exposure [7]. To attenuate this noise, a dynamic high pass filter, filtering low amplitude components in the Fourier space, is applied.

After the reconstruction of the jet image, the spreading angle of the jet core can be measured. For this measurement, several columns of the reconstructed image, located just behind the jet head, are selected (figure 7). Then the averaged curve of pixel intensity of the columns is obtained (green curve in figure 8). In order to find the jet core, a special criterion is defined according to the derivative curve, based on the fact that, in the vicinity of the jet core, derivative is high because of sharp variations in pixel intensity. So the boundary of jet core is conventionally assumed to be bounded by the highest variations of intensity. In figure 8 the dashed lines show the places of highest derivatives in both sides of the 
jet and the distance between these vertical lines represents the jet core width. Then, the spreading angle can be obtained by comparison with a similar measurement of the jet core width at the nozzle exit.

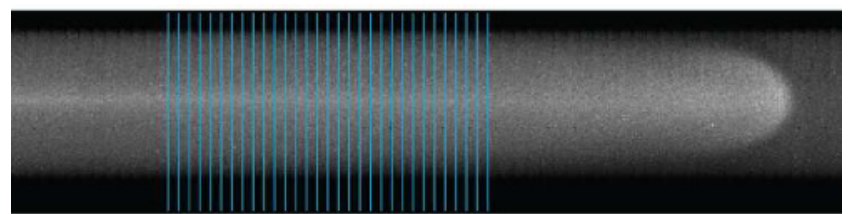

Fig. 7. Lateral selected columns of reconstructed image, behind the jet head

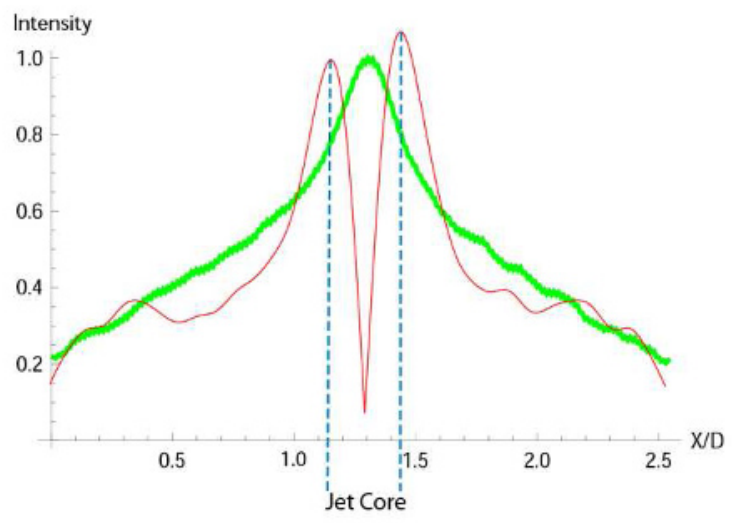

Fig. 8. Green curve: averaged curve of pixel intensity, Red curve: derivative of green curve, absolute value

\section{Results}

Here one example of jet development is reported showing the application of the present image processing methods to jet display and structural velocities measurement. The jet under test is a heavy jet of Xenon in Argon, travelling at Mach 15, and its development is shown in figure 9.

Part 1: $t<10 \tau$

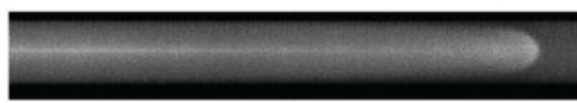

Part2: $30 \tau<\mathrm{t}<34 \mathrm{\tau}$

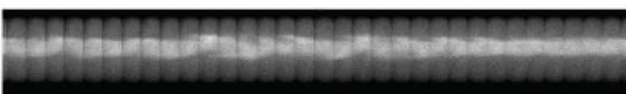

Part3: $34 \tau<t<37 \pi$

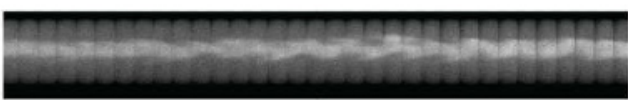

Fig] 9. Development of a Xenon jet in Argon ambient, Jet direction: left to right. Time scale $\tau=2 \mathrm{~ms}$ (jet radius at the nozzle exit over the sound speed inside the jet)

By means of measured velocities, groups of subsequent images are used to compose partial jet reconstructions, i.e. partial superposition of frames based on the local velocity of the relevant structures. The first part of figure 9 is the free jet, before the impact to the vessel end. In the second part the flow develops weak $\mathrm{K}-\mathrm{H}$ instabilities, driven by the shear between the jet core and the surrounding backward flow caused by jet head impact. This instability does not spoil the axial symmetry of the fow for a while, but later produces a stronger perturbation, which non-linearly interacts with the outer fow. This can be clearly seen in the third part. These very strong instabilities have a variable wavelength changing with instability growth and contribute to the formation of the complex internal jet structure. The velocities of fow structures have been measured by means of correlation techniques applied to the pixel matrices of consecutive images.

The head velocity is measured easily and is about $115 \pm 20$ $\mathrm{m} / \mathrm{s}$. The instability wave velocities in part 2 and 3 are about $370 \pm 20 \mathrm{~m} / \mathrm{s}$. For the flow between jet head and large instabilities, where the jet resembles a collimated beam, a correlation velocity is interpolated by other velocities and is variable between 250 and $300 \mathrm{~m} / \mathrm{s}$. Spreading angle of this free jet, in the first part of figure 9 , is about 0.8 degree.

\section{Conclusions}

Several image processing techniques have been applied to the study of hypersonic jets morphology. Wide images of the jets were reconstructed by partial superposition of de-noised frames. The reconstructions have been made possible by the use of an accurate technique to measure flow structures velocities. Finally, the same images have been analyzed in such a way as to extract measurements of spreading angles.

The sample results demonstrate that the proposed methods are successful and effective to display the jet flow morphology.

\section{References}

1. $3[\$ \square R o s e n, G$. Hughes, D. Comer, P.E. Hardee in: A comparison of the morphology and stability of relativistic and nonrelativistic jets, Astrophysical Journal, 516 (1999)

2. R. Presura, S. Neff, L. Wanex, in: Experimental Design for the Laboratory Simulation of Magnetized Astrophysical Jets, Astrophys Space Sci 307(2007)

3. C. D. Gregory, J. Howe, B. Loupias, S. Myers, M.M. Notley, Y. Sakawa, A. Oya, R. Kodama, M. Koenig and N. C. Woolsey N C, in: Astrophysical Jet Experiments with Colliding Laser-produced Plasmas, ApJ 676420 (2008)

4. D. J. Ampleford, S. V. Lebedev, A. Ciardi, S. N. Bland, S. C. Bott, G. N. Hall, N. Naz, C. A. Jennings, M. Sherlock, J. P. Chittenden, A. Frank, E. Blackman, in: Laboratory Modeling of Standing 
Shocks and Radiatively Cooled Jets with Angular Momentum, Astrophys Space Sci 307 (2007)

5. P. Hartigan, J. M. Foster, B. H. Wilde, B. F. Coker, P. A. Rosen, J. F. Hansen, B. E. Blue, R. J. R. Williams, R. Carver and A. Frank, in: Laboratory Experiments, Numerical Simulations, and Astronomical Observations of Deflected Supersonic Jets: Application to HH110, ApJ 7051073 (2009)

6. M. Belan, Apparato a fascio elettronico per visualizzazioni e misure su getti di gas a bassa pressione, Scientific Report DIA SR-12-03, Politecnico di Milano, Dipartimento di Ingegneria Aerospaziale (2012)

7. C. Tropea, A. L. Yarin, J. F. Foss, Springer Handbook of Experimental Fluid Mechanics (2007) 